\title{
An intervention study to promote self-improvement of lifestyle in a Japanese community: a new health support program
}

\author{
Kumiko Fukumoto Chang-nian Wei $\cdot$ Hiroshi Matsuo $\cdot$ Koichi Harada \\ Shi-chen Zhang $\cdot$ Luyinga Kalay $\cdot$ Takeshi Yamashiro $\cdot$ Takeshi Nishikawa • \\ Eiichi Araki $\cdot$ Atsushi Ueda
}

Received: 8 March 2010/Accepted: 8 November 2010/Published online: 18 January 2011

(C) The Japanese Society for Hygiene 2011

\begin{abstract}
Objective To assess the effectiveness of two health support programs developed to improve the lifestyle of community residents through exercise, nutrition-based health education, and group dynamics.

Methods A total of 171 subjects were selected from community residents showing an abnormal result in at least one metabolic syndrome-related laboratory test. The subjects were divided into two groups: a group participating only in the 5-month initiation program (i.e., the initial program; $n=83$ ), and a group participating both the initiation program and the subsequent 6-month enhancing program (i.e., the total program; $n=88$ ). Each group was followed up for 1 year after completing the intervention program. The effectiveness of the intervention was determined based on data from the Health Promoting Lifestyle Profile II and laboratory tests, which were collected four times during the 2-year follow-up. Thirty-nine individuals were selected as the control group, based on the same
\end{abstract}

C. Wei $\cdot$ K. Harada $\cdot$ S. Zhang $\cdot$ L. Kalay $\cdot$ A. Ueda

Department of Preventive and Environmental Medicine,

Graduate School of Medical Sciences, Kumamoto University,

Kumamoto, Japan

H. Matsuo

Kumamoto Wellness Support Institute, Kumamoto, Japan

T. Yamashiro - T. Nishikawa $\cdot$ E. Araki

Department of Metabolic Medicine, Graduate School of Medical

Sciences, Kumamoto University, Kumamoto, Japan

K. Fukumoto $(\square)$

Department of Nursing, Kyushu University of Nursing

and Social Welfare, 888 Tomio, Tamana,

Kumamoto 865-0062, Japan

e-mail: fukumoto@kyushu-ns.ac.jp criteria, to confirm the effectiveness of completing the intervention program.

Results The results demonstrate that completion of the initiation programe was an effective intervention in terms of individual improvements in lifestyle and laboratory test results. The improvements achieved by the end of the initiation program had been sustained at the end of the total program, and were similar in both groups. One year after the end of the total program, both groups showed similar findings, confirming the effectiveness of the intervention. Conclusion The results of this study demonstrate the effectiveness of two health support programs for improving the lifestyle of community residents. It is worth noting that, at the end of the 2-year follow-up, the improvements in lifestyle due to the initiation program were similar to those of the total program.

Keywords Intervention study $\cdot$ Lifestyle $\cdot$ HealthPromoting Lifestyle Profile-II · Health support program · Metabolic syndrome

\section{Introduction}

In 1990, the National Center for Health (Atlanta, GA) reported that heart disease, malignant neoplasm, and cerebrovascular disease were the three leading causes of death in the USA [1]. McGinnis and Foege subsequently demonstrated that about $50 \%$ of all cases of these diseases were attributable to the lifestyle [2]. Further, in 2002, the World Health Organization (WHO) [3] stated that the importance of preventive measures for cardiovascular disease and the need to reduce unhealthy diets and physical inactivity should be emphasized as its new global health policy. 
The Japanese Ministry of Health, Labour and Welfare reported in Vital Statistics 2004 that approximately 60\% of causes of death in Japan were attributed to lifestyle-related diseases [4]. In addition, according to the Patient Survey 2002 [4], among the 7.93 million patients registered in the survey, $88 \%$ were suffering from hypertensive disease, $29 \%$ from diabetes mellitus, $17 \%$ from cerebrovascular disease, $16 \%$ from malignant neoplasm, and $12 \%$ from ischemic heart disease. The above five diseases, with the exception of malignant neoplasm, are known to be closely related to arteriosclerosis caused by lifestyle conditions involving multiple risk factors. The complication of such multiple factors, reported as syndrome X [5], the deadly quartet [6], visceral fat syndrome [7], metabolic syndrome (MS), or multiple risk factor syndrome [8], has been proven to increase the risk of arteriosclerosis. Suka et al. [9] reported that the accumulation of risk factors related to metabolic syndrome may increase in the presence of such diseases as obesity and diabetes mellitus, and they pointed out the need for preventive intervention in the pre-metabolic syndrome stages.

There have been a number of lifestyle studies on community residents. The first study to clarify a correlation between health and lifestyle was the follow-up study by Berkman and Breslow [10]. In Japan, Morimoto et al. studied the link between lifestyle and cancer prevention as well as physical, mental, and genetic health based on more spiritual views of life and health. In their report, they also define lifestyle in terms of an integration of health awareness and the value and view of life in each community [11]. In the Ottawa Charter of Health Promotion (1986), the WHO defines lifestyle not only in terms of health-promoting behavior but also in terms of the quality of life (QOL), such as health awareness and satisfaction [12].

This new concept of lifestyle indicates that, in order to promote lifestyle as a means for achieving improved health, a system is needed in the community setting which helps each resident of that community to develop a suitable lifestyle by providing knowledge, techniques, and a practical method of health education.

Pender presented a health-promoting model as a conceptual framework that nurtures the aforementioned health behavior [13]. Walker et al. [14] proposed a health-promoting lifestyle with a rating scale, which they developed as a questionnaire, named the Health Promoting Lifestyle Profile-II (HPLP-II). Each item on the questionnaire consists of concepts involving a multidimensional pattern of self-initiated actions and perceptions that serve to maintain or enhance the level of individual wellness, self-actualization, and fulfillment. HPLP-II can determine the value of health-promoting behavior as an index of the lifestyle by scoring each item. The Japanese version of HPLP-II was developed by Wei et al. [15].
A number of studies on lifestyle involving the perception of self-actualization have been reported from abroad using HPLP-II [16, 17]. In Japan, there has been one published study on the relationship between medical observations, including obesity, and lifestyle factors, such as physical activity or dietary habits [11]. However, no empirical follow-up study of community-based intervention(s) has been reported that assesses the relationship between metabolic syndrome-related test findings and the overall lifestyle as a fundamental condition of the QOL. The results of this study, however, indicated that the concept and techniques of health promotion proposed by the WHO [12] may be the most effective way for community residents to achieve lifestyle-related self-control through administrative or regional health activities.

Based on the above findings of lifestyle studies, we have developed a health support program to improve individual lifestyle and enhance the QOL of each subject, resulting in the prevention of metabolic syndrome. The program is characterized by exercise, nutrition-based health education, and group dynamics. The aim of the study reported here was to demonstrate the effectiveness and validity of the intervention program for the self-oriented improvement of lifestyle, using HPLP-II as an evaluation tool to determine subjects' lifestyle scores.

\section{Methods}

Subjects

In 2002, 2,986 adults, aged 30-69 years, among the 31,541 residents of Town A, Kumamoto, Japan, underwent the periodic community-based health checkup required by Japanese law ("Health and Medical Service Law for the Aged"; passed in 1977). We screened of these, 417 individuals had at least one of the MS criteria and thus eligible for enrollment in our study (Table 1). Individuals who took medication for hypertension, dyslipidemia, or diabetes were subsequently excluded. The remaining subjects were requested by mail to provide informed consent prior to enrollment. Ultimately, 103 subjects $(24.7 \%)$ provided consent and were enrolled.

In 2003, 3,627 adults, all of the same age, among the 31,489 residents of the same town were subjected to the same selection process. Ultimately, 101 subjects $(20.0 \%)$ from among 505 eligible individuals consented to participate in the study. The total number of subjects in the intervention group was therefore $204(22.1 \%)$. An additional 39 residents of Town A were selected as a control group in order to clarify the effectiveness of the intervention program; the selection of these individuals was based on the same criteria and procedure as that for the intervention group. 
Table 1 Inclusion criteria

\begin{tabular}{ll}
\hline Metabolic syndrome components & Value \\
\hline Body mass index & $\geqq 25$ \\
Systolic blood pressure & $\geq 130$ to $<160 \mathrm{mmHg}$ \\
Diastolic blood pressure & $\geq 85$ to $<100 \mathrm{mmHg}$ \\
Fasting blood glucose & $\geq 110$ to $<126 \mathrm{mg} / \mathrm{dl}$ \\
HbA1c & $\geq 5.5$ to $<6.1 \%$ \\
Triglycerides & $\geq 150 \mathrm{mg} / \mathrm{dl}$ \\
HDL cholesterol & $<40 \mathrm{mg} / \mathrm{dl}$ (males); \\
& $<50 \mathrm{mg} / \mathrm{dl}$ (females) \\
LDL cholesterol & $\geq 140 \mathrm{mg} / \mathrm{dl}$ \\
Total cholesterol & $\geq 220 \mathrm{mg} / \mathrm{dl}$
\end{tabular}

$H b A 1 c$ Glycosylated hemoglobin, $L D L$ low-density lipoprotein, $H D L$ high-density lipoprotein

The intervention subjects who satisfied at least one of the above criteria based on the laboratory test data were assessed as having premetabolic syndrome

Outline of the intervention follow-up study

Two series of a health support programs were developed. The design of the intervention study is shown in Fig. 1.

The "initiation program" comprised seven lifestyle intervention courses that were given by diabetologists, trained nurses, dietitians, and physical therapists who understood the aims of the study. All 204 subjects attended these courses over a 5-month period. Five of the seven courses (1st, 2nd, 4th, 5th, and 7th) comprised group lectures, and the remaining two (3rd and 6th) comprised one-on-one counseling sessions. Five lectures focused on MS, nutrition, exercise, and behavioral modifications. After the second and seventh lectures, the subjects were divided into small groups of five to eight people for 30-min group discussions. The aim of the group discussions was to enable each individual to draw on and learn from the experiences of other people in the group and also to provide each individual with mutual encouragement to maintain the recommended lifestyle modifications. In course 3 (counseling session), data from the health check-up and questionnaire were used by the health professionals to establish specific goals with each individual that would result in an improvement of. In course 6, the established goals for each individual were reviewed and reset, with input from public health nurses and dietitians.

Upon completion of the initiation program, all 204 subjects were classified into two groups: the initiation and total groups. The procedure of assigning the subjects to one of the two groups was as follows: (1) subjects were divided into males and females and arranged according to increasing age; (2) subjects in each sex group were alternately allocated to the two groups in order of age. This procedure resulted in two groups with a similar age distribution: a group participating in only the initiation program, named the initiation group, and a group participating in the initiation program and the subsequent 6-month enhancing program, i.e., the total program, named the total group.

The enhancing program for the total group comprised six courses, with various experience-based learning opportunities involving exercises, and was based on the method of once-a-month group education. The aim of the program was to persuade the subjects to experience the pleasure of exercise and to become able to choose exercise modes that suited their lifestyle. In addition, they subjects were provided with a daily calendar on which specific numerical data was recorded for the self-assessment of the effectiveness of their activities, and advice was listed regarding the frequency of exercises, such as stretching, muscle workout, aerobic exercise, etc.

Thus, to summarize, the subjects were provided with one or two series of health support programs, respectively, aimed at the self-oriented improvement of the lifestyle of the community residents: one was the 5-month intervention of the initiation program, and the other was the 11-month intervention of the initiation plus enhancing program. During the 6-month enhancing program, the subjects in the initiation group were left to control each lifestyle factor by themselves and only received a leaflet containing general information about voluntary exercise.

There was a gym and swimming pool in the municipal health promotion center, and the subjects, including the control group, could use these facilities free of charge during the entire intervention period.

The subjects were allocated into one of the two program series by an independent staff member of the current project in Town A. None of the subjects and none of the corresponding project researchers were aware of the group allocation until completion of the initiation program.

\section{Measurements}

Data were collected four times during the 2-year follow-up following completion of each intervention program, namely, "Baseline", "Interim", "Final", and "A-yearafter". In each assessment step, the subject' lifestyle based on the HPLP-II score and pre-metabolic syndrome-related laboratory test data were determined.

The HPLP-II $[14,15]$ comprises 52 items categorized into six subscales. Each item has a 4-point score format (from 1 to 4 ) assessing daily physical and spiritual activities. The six subscales represent health responsibility (HR), spiritual growth (SG), physical activity (PA), interpersonal relations (IR), nutrition (N) and stress management (SM).

The determinants of medical data related to metabolic syndrome are as follows: body mass index (BMI), fasting blood glucose (FBG), triglycerides (TG), total cholesterol 
"Baseline assessment"

Mode of practice

\begin{tabular}{|l|c|}
\hline \multicolumn{2}{|c|}{ Intervention Group (204 subjects) } \\
\hline Initiation group (102) & Total group (102) \\
\hline
\end{tabular}

Initiation program
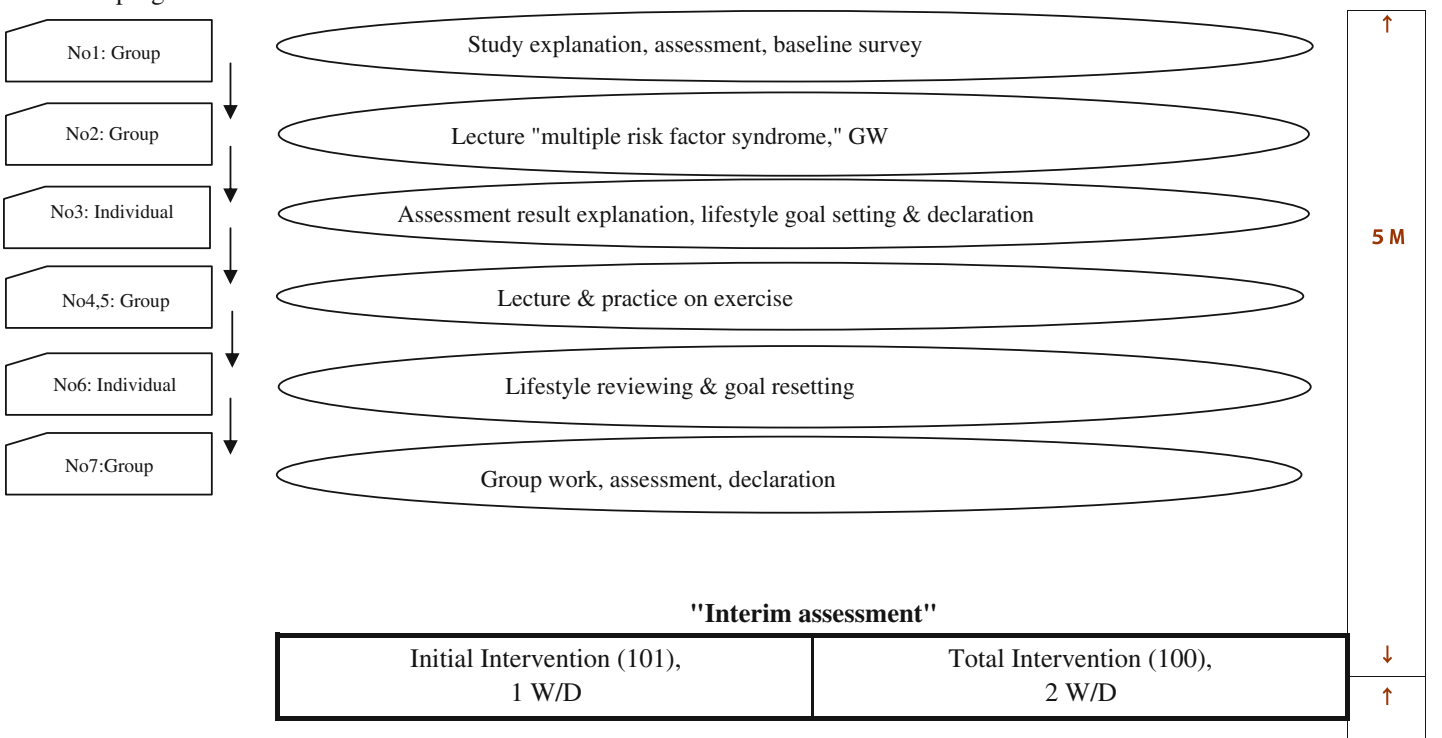

Enhancing program
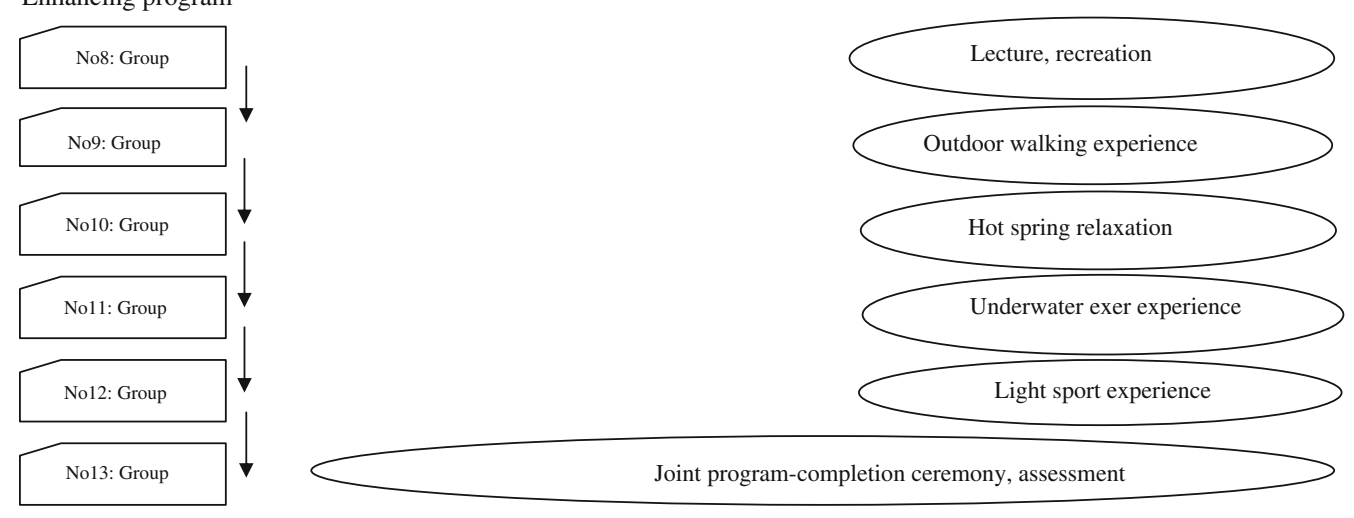

\begin{tabular}{|c|c|} 
"Final assessment" \\
\hline \begin{tabular}{|c|c|}
\hline Initial Intervention (100), & Total Intervention (96), \\
2 W/D & 6 W/D \\
\hline
\end{tabular}
\end{tabular}

Control Group (39)

"A-year-after-assessment"

\begin{tabular}{|c|c|}
\hline $\begin{array}{c}\text { Initial Intervention (83), } \\
19 \mathrm{~W} / \mathrm{D}\end{array}$ & Total Intervention (88), \\
\hline
\end{tabular}

Initiation Intervention:5-Month-Intervention,Total Intervention:11-Month-Intervention,W/D;withdrawn

Fig. 1 Outline of study design

(T-C), HDL-cholesterol (HDL-C), systolic blood pressure (SBP), and diastolic blood pressure (DBP) (Table 1). The data were obtained from the community-based periodic health check-ups in Town A, as mentioned above.
Statistical analysis

The effectiveness of the initiation and total programs was assessed using the paired $t$ test to determine the difference in 
the mean scores overall and for the six subscales of HPLP-II and laboratory test values for each group. Bonferroni correction [18] was used for multiple comparison testing.

The scores of the initiation and total groups were compared and analyzed using the unpaired $t$ test (test for two independent samples).

Statistical analysis was performed employing SPSS ver, 11.0J for Windows (SPSS, Chicago, IL).

Ethical considerations

During the recruitment process, all eligible participants, including those in the control group, were informed of the purpose of the study and provided advice on the benefits of an appropriate caloric intake and increasing physical activity. All of the subjects, including the control group, were guaranteed that they were free from any burden or disadvantage due to their participation non-participation.

This study was designed and conducted under the approval of the Japanese Ministry of Health, Labour and Welfare through a national model project, "Health-Up model projects of National Health Insurance".

\section{Results}

The trend in subject dropout during the 2-year followup study and comparison of baseline scores between the intervention and dropout groups

By similarly employing the random allocating procedure, the 204 subjects (64 males and 140 females) were divided into the initiation and total groups, respectively, with 102 subjects in each, prior to completion of the initiation program (Table 2). As shown in Fig. 1, three subjects (1 from the initiation group and 2 from the total group) dropped out during the initiation program, and eight subjects ( 2 from the initiation group and 6 from the total group) dropped out during the subsequent enhancing program. During the first follow-up year after completion of the total program, 25 subjects (17 from the initiation group and 8 from the total group), dropped out. In total, 33 subjects, denoted the "dropout group" [19 from the initiation group (18.6\% of the initial subjects) and 14 from the total group (13.7\%)] dropped out during the entire 2-year follow-up. There was no significant difference in dropout rate between the two groups. There was no significant gender-associated difference in dropout rate: 13 males $(20.3 \%$ of the initial male subjects) versus 20 females (14.3\% of the initial female subjects).

Seven participants dropped out due to personal illness or the need to care for sick family members. The remaining 26 dropouts missed the routine health checkup held 1 year after the total program. A total of 171 subjects completed the 2-year follow-up: 83 of the initiation group and 88 of the total group.

Among the 204 subjects, there was no significant difference in the sex ratio between the initiation, total, and dropout groups. In contrast, there was a significant difference $(p<0.05)$ between the sex ratio in the control group and that in the intervention group. There was a significant difference $(p<0.01)$ in age between the initiation, total, and dropout groups $(61.9 \pm 5.4$ vs. $61.8 \pm 5.4$ vs. $58.4 \pm 6.8$, respectively. The age of the control group was $61.8 \pm 6.6$, which was not significantly difference from that of the intervention group.
Table 2 Sex and age composition of the intervention (initiation and total), dropout, and control groups
Initial 5-month-intervention, Total 11-month-intervention, Control no intervention

\begin{tabular}{lllccc}
\hline \multirow{2}{*}{$\begin{array}{l}\text { Sex } \\
\text { Age } \\
\text { (years })\end{array}$} & \multicolumn{2}{c}{ Intervention (all cases) } & $\begin{array}{l}\text { Control, } \\
n(\%)\end{array}$ \\
\cline { 2 - 6 } Male & Initiation, $n(\%)$ & Total, $n(\%)$ & Dropout, $n(\%)$ & $2(28.6)$ \\
& $40-49$ & $0(0.0)$ & $1(3.6)$ & $2(15.4)$ & $3(42.9)$ \\
& $50-59$ & $4(17.4)$ & $4(14.3)$ & $4(30.8)$ & $0(0.0)$ \\
& $60-64$ & $11(47.8)$ & $13(46.4)$ & $4(30.8)$ & $2(28.6)$ \\
& $65-69$ & $8(34.8)$ & $10(35.7)$ & $3(23.1)$ & $7(100.0)$ \\
& Subtotal & $23(100.0)$ & $28(100.0)$ & $13(100.0)$ & $1(3.1)$ \\
& $40-49$ & $2(3.3)$ & $3(5.0)$ & $4(20.0)$ & $8(25.0)$ \\
& $50-59$ & $13(21.7)$ & $15(25.0)$ & $5(25.0)$ & $10(31.3)$ \\
& $60-64$ & $21(35.0)$ & $21(35.0)$ & $9(45.0)$ & $32(100.0)$ \\
& $65-69$ & $24(40.0)$ & $21(35.0)$ & $2(10.0)$ & $3(7.7)$ \\
& Subtotal & $60(100.0)$ & $60(100.0)$ & $20(100.0)$ & $11(28.2)$ \\
& $40-49$ & $2(2.4)$ & $4(4.5)$ & $6(18.2)$ & $10(25.6)$ \\
& $50-59$ & $17(20.5)$ & $19(21.6)$ & $9(27.3)$ & $15(38.5)$ \\
& $60-64$ & $32(38.6)$ & $34(38.6)$ & $13(39.4)$ & $39(100.0)$ \\
\hline
\end{tabular}


Table 3 Baseline of Health Promoting Lifestyle Profile-II scores and laboratory test values of the intervention (Initial + total), dropout, and control groups

\begin{tabular}{|c|c|c|c|c|c|}
\hline & $\begin{array}{l}\text { Intervention } \\
(n=171)\end{array}$ & $\begin{array}{l}\text { Dropout } \\
(n=33)\end{array}$ & $\begin{array}{l}\text { Differences: } \\
\text { intervention } \\
\text { and dropout }\end{array}$ & $\begin{array}{l}\text { Control } \\
(n=39)\end{array}$ & $\begin{array}{l}\text { Differences: } \\
\text { intervention } \\
\text { and control }\end{array}$ \\
\hline Total HPLP & $2.67 \pm 0.36$ & $2.57 \pm 0.32$ & ns & $2.67 \pm 0.33$ & $\mathrm{~ns}$ \\
\hline $\mathrm{HR}$ & $2.47 \pm 0.48$ & $2.34 \pm 0.50$ & $\mathrm{~ns}$ & $2.58 \pm 0.43$ & $\mathrm{~ns}$ \\
\hline PA & $2.02 \pm 0.67$ & $1.70 \pm 0.61$ & $*$ & $1.91 \pm 0.54$ & $\mathrm{~ns}$ \\
\hline $\mathrm{N}$ & $2.93 \pm 0.35$ & $2.86 \pm 0.38$ & ns & $2.95 \pm 0.34$ & $\mathrm{~ns}$ \\
\hline SG & $2.68 \pm 0.49$ & $2.74 \pm 0.43$ & $\mathrm{~ns}$ & $2.75 \pm 0.58$ & $\mathrm{~ns}$ \\
\hline IR & $3.05 \pm 0.46$ & $2.95 \pm 0.51$ & ns & $2.98 \pm 0.48$ & ns \\
\hline SM & $2.80 \pm 0.49$ & $2.73 \pm 0.39$ & ns & $2.81 \pm 0.40$ & $\mathrm{~ns}$ \\
\hline BMI & $23.7 \pm 3.2$ & $24.5 \pm 2.9$ & ns & $23.6 \pm 2.7$ & $\mathrm{~ns}$ \\
\hline SBP & $130.2 \pm 17.8$ & $124.1 \pm 14.5$ & ns & $128.7 \pm 14.1$ & ns \\
\hline DBP & $77.9 \pm 10.2$ & $75.7 \pm 9.9$ & ns & $76.1 \pm 9.7$ & ns \\
\hline $\mathrm{TC}$ & $224.2 \pm 32.6$ & $208.6 \pm 30.8$ & $*$ & $222.0 \pm 29.5$ & $\mathrm{~ns}$ \\
\hline TG & $126.8 \pm 74.2$ & $123.9 \pm 56.2$ & ns & $136.6 \pm 91.3$ & $\mathrm{~ns}$ \\
\hline HDL-C & $60.2 \pm 14.4$ & $59.6 \pm 15.5$ & ns & $62.0 \pm 14.1$ & $\mathrm{~ns}$ \\
\hline FBG & $77.6 \pm 14.6$ & $77.3 \pm 11.4$ & ns & $87.3 \pm 10.2$ & $* * *$ \\
\hline
\end{tabular}

$*_{p}<0.05, * * * p<0.001$, according to the unpaired $t$ test

All values are given as the mean $\pm \mathrm{SD}$

$H R$ health responsibility, $P A$ physical activity, $N$ nutrition, $S G$ spiritual growth, $I R$ interpersonal relations, $S M$ stress management, $B M I$ body mass index, $S B P$ systolic blood pressure, $D B P$ diastolic blood pressure, $T C$ total cholesterol, $T G$ triglycerides, $H D L-C$ HDL cholesterol, $F B G$ fasting blood glucose, HPLF Health Promoting Lifestyle Profile-II, Total HPLP total average of HPLP, ns not significant

The HPLP-II scores and results of laboratory tests for all subjects at baseline are shown in Table 3. The score of each subscale of HPLP-II was generally higher in the intervention than in the dropout group, with the exception of SG. However, a significant difference was noted only for PA $(p<0.05)$.

Concerning the laboratory test data, the value of T-C $(p<0.05)$ was significantly higher in the intervention than in the dropout group.

The scores of HPLP-II and the laboratory test data showed no significant difference between the intervention and control groups except for FBG.

\section{Results of the Interim assessment}

The scores of HPLP-II by each subscale and values of laboratory tests in the intervention and control groups during the four periods of assessment are shown in Tables 4 and 5 .

As shown in Table 4, the score for each subscale of the HPLP-II in the intervention group at the interim was significantly higher than that at baseline $(p<0.01$ per item). The same tendency of improvement was shown in both the initiation and total groups, with the exception of IR, for which there was no significant difference in the score of each HPLP-II subscale between both groups.
As shown in Table 5, significantly lower values of BMI $(p<0.01)$ and TG $(p<0.05)$ and significantly higher values of HDL-C $(p<0.01)$ were present at the interim than at baseline. However, significantly higher values of DBP and FBG were seen at the interim than at baseline.

\section{Results of the final assessment}

As shown in Table 4, in the initiation group, the scores for all of the HPLP-II subscales were lower at the final assessment period than at the interim assessment, with a particularly significant score reduction in PA $(p<0.001)$; however, all subjects maintained significantly higher scores compared to the baseline, with the exception of IR. In the total group, a small change was noted in the score of each subscale between the interim and final assessment, with the exception for PA. The score for PA in the total group at the final assessment was significantly higher than that in the initiation group $(p<0.01)$.

With respect to the control group, a small change was noted in all HPLP-II subscales between the baseline and final assessment. The control group showed a tendency toward lower scores in all HPLP-II subscales compared to the intervention group, especially for PA and $\mathrm{SM}$ ( $p<0.01$ per each).

As shown in Table 5, in the initiation group, a significant improvement in laboratory test values was seen in 
Table 4 The scores of HPLP-II in the intervention and control groups

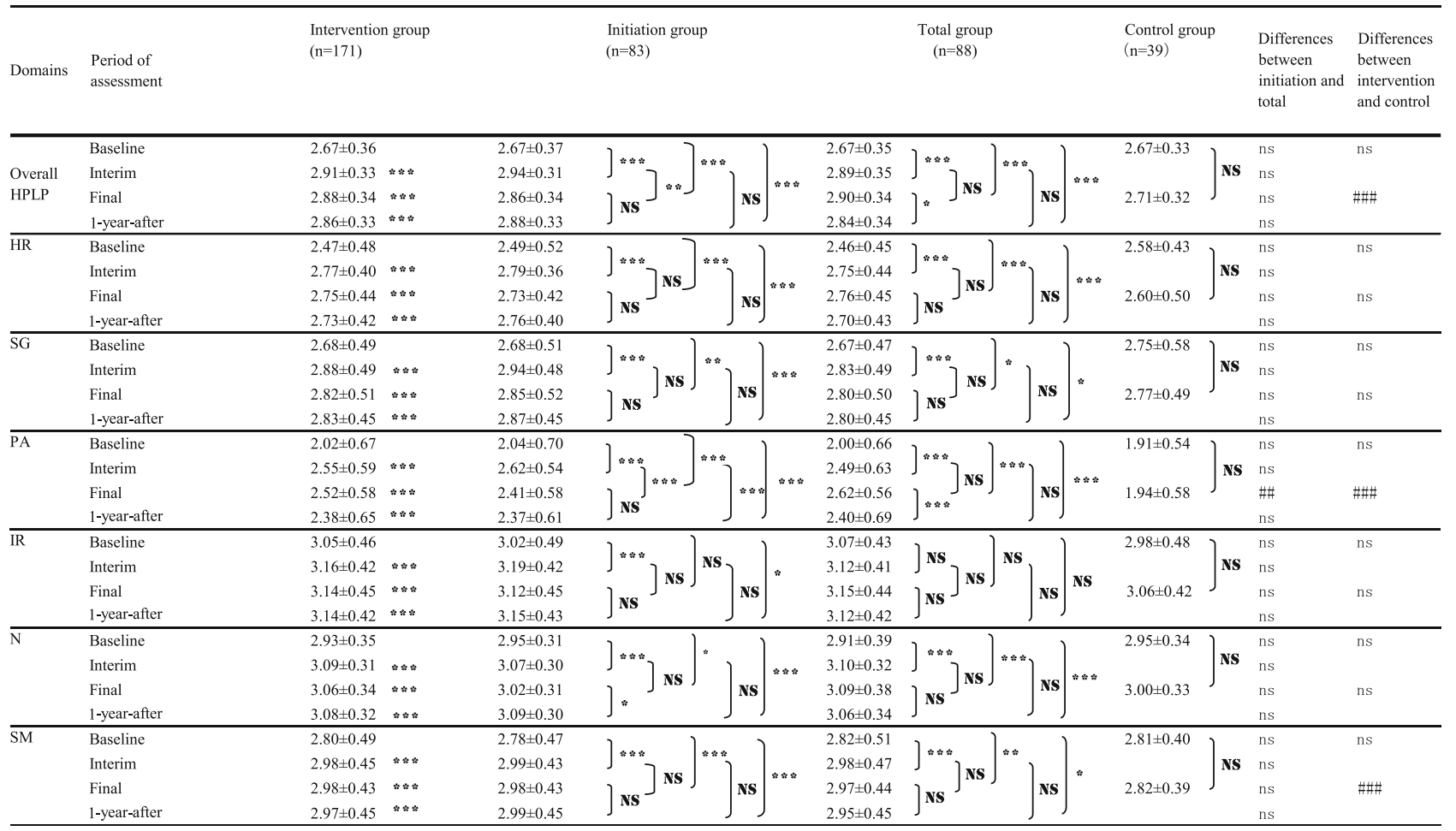

Domains of HPLP II: see Table 2

Baseline assessment at the beginning of intervention, Interim at the completion of the initiation program, Final at the completion of the total program, 1-year-after at the end of the two-year follow-up

$* * * p<0.001, * * p<0.01, * p<0.05$ by paired $t$ test (Bonferroni correction)

\#\#\# $p<0.001$, \#\# $p<0.01$, \# $p<0.05$ by unpaired $t$ test

All values are given as the mean $\pm \mathrm{SD}$

SBP $(p<0.01)$, TG $(p<0.01)$, and HDL-C $(p<0.01)$ between the baseline and final assessment. A similar tendency was seen in the total group, with a significant improvement observed for BMI $(p<0.01)$, SBP $(p<0.001)$, TC $(p<0.05)$, and HDL-C $(p<0.001)$. In general, more favorable values were seen in the total group than in the initiation group, with the most notable of these being a significantly lower TG value in the latter.

In the control group, there was no significant change between the baseline and final assessment. FBG was significantly higher in the control group than in the intervention group.

Results of the 1-year-after assessment

In the initiation group, higher scores were generally noted in each HPLP-II subscale between the final and 1-yearafter assessment, especially for $\mathrm{N}(p<0.05)$, with the exception of PA. Compared with the baseline, significantly higher scores for all HPLP-II subscales were seen at the 1 -year-after assessment $(p<0.01$ and 0.05$)$. In terms of the total group, however, lower scores were generally seen in all HPLP-II subscales at the 1-year-after assessment than at the final assessment, especially overall $(p<0.05)$ and for PA $(p<0.001)$. Relative to the baseline values, all the scores for all HPLP-II subscales were significantly higher at the 1 -year-after assessment $(p<0.001$ and $p<0.05)$, with the exception of IR.

There was no significant difference in each score of the HPLP-II subscales between the initiation group and total group at the 1-year-after assessment.

In the intervention group, significantly higher scores were also seen in all of the HPLP-II subscales $(p<0.001$ per each) between the 1-year-after assessment and baseline assessment.

As shown in Table 5, in the initiation group, the laboratory values for BMI showed significant improvement $(p<0.05)$ and those for FBG were significantly lower $(p<0.001)$ between the 1-year-after and final assessments. Compared with the baseline, improvements were generally seen in all of the items, especially BMI $(p<0.01)$ and DPB $(p<0.01)$, with the exception of FBG $(p<0.001)$. 
Table 5 The laboratory test values in the intervention and control groups

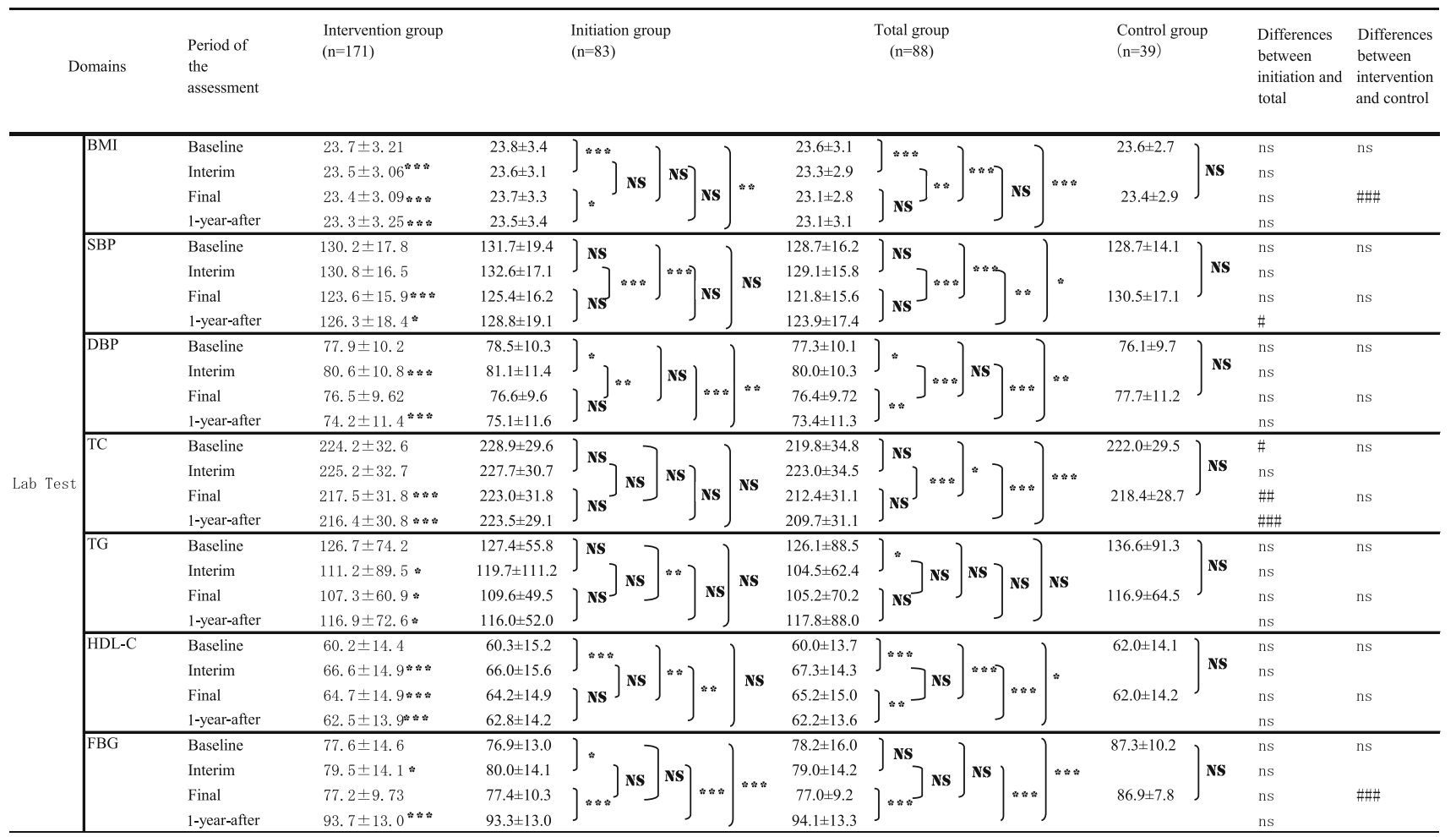

Domains of laboratory test: see Table 2

Baseline assessment at the beginning of intervention, Interim at the completion of the initiation program, Final at the completion of the total program, 1-year-after at the end of the two-year follow-up

$* * * p<0.001, * * p<0.01, * p<0.05$ by paired $t$ test (Bonferroni correction)

\#\#\# $p<0.001$, \#\# $p<0.01$, \# $p<0.05$ by unpaired $t$ test

All values are given as the mean $\pm \mathrm{SD}$

For the total group, a significant improvement was seen in DBP $(p<0.01)$ and significantly lower values were noted in HDL-C $(p<0.01)$ and FBG $(p<0.001)$ between the 1-year-after and final assessments. Compared with the baseline, a similar tendency was noted in the total and initiation groups; in particular, there was a significant improvement in BMI $(p<0.01)$, SBP $(p<0.001)$, TC $(p<0.05)$, and HDL-C $(p<0.001)$. In general, more favorable values were noted in the total than in the initiation group, with especially significantly more favorable values for SBP $(p<0.05)$ and TC $(p<0.001)$.

For the intervention group, significant improvements were seen in the 1-year-after compared to the baseline assessment $(p<0.05-0.001)$, with the exception of FBG $(p<0.01)$.

\section{Discussion}

As earlier published reports on health promotion [12] and health education [19] have demonstrated, the most effective means to improve lifestyle with the aim of disease prevention, such as MS, one of the more urgent health conditions associated with community health, is to encourage each individual to nurture a self-oriented ability to improve and maintain an appropriate lifestyle.

Accordingly, we have developed a series of two health support programs. The first program, the initiation program, provides participants with fundamental information on lifestyle-related diseases, such as MS, and promotes the concept of self-improvement with respect to each lifestyle factor by means of individual and/or group instruction by specialists in their field. The program is characterized by exercise and nutrition-based health education, partly in the context of group dynamics. The second program, the enhancing program, provides participants with technical ways to employ available exercises, including the effective usage of hot springs, with the aim of persuading the subjects to experience the pleasure of exercise and become able to choose exercise suited to their lifestyle. In this program, participants are provided with a daily calendar for health improvement on which specific numerical data to 
facilitate the self-assessment of the effectiveness of their activities can be recorded.

Prior to the study, we hypothesized that it would be more effective to receive both the initial and enhancing programs, i.e., the total program, than to only participate in the initial program. We then conducted the study reported here to determine which of these two approaches, the initiation program or the total program, would be more effective among our subjects for improving lifestyle.

In general, an intervention-based follow-up study such as the one reported here may involve fundamental issues that affect the interpretation of the results. One such factor is the effect of experience on refraining from replying to the questionnaire [20]; the second is the effect of a regression to the mean [20].

To reduce these biases, we divided the subjects into two groups, initiation and total, by employing a method similar to the randomization of subjects-closely resembling a double blind method-and compared the scores between the two groups at four points in time using the paired $t$ test. We also designated a control group to clarify the effectiveness of the intervention program at baseline and the final assessment, i.e., a crossover design [20]. As shown in Tables 4 and 5, there was no significant difference in the scores of all items at baseline, with different scores between the two intervention groups and control group at the interim and final assessments. These results suggest that our procedures may have reduced the bias due to removing the experience of completing the same questionnaire and the effect of regression to the mean.

We demonstrated that the initiation program was effective in improving the lifestyles of individuals and in sustaining this state of self-improvement for 1 year after the completion of the intervention; this is the same as that obtained for the total program. The results suggest that both our programs, initiation and total, were effective in educating community residents whose test findings indicated the presence of pre-metabolic syndrome, thereby necessitating a certain degree of management. At the 1-year follow-up after the end of enhancing intervention, both the total and initiation groups showed the favorable effects of the initiation program, albeit at slightly lower levels than the respective levels obtained at the end of the initiation program. The improvements observed at the 1-year followup in both groups were significant in comparison with the baseline values. In other words, the effect of the 5-month initiation program was sustained to a satisfactory level 1 year after the end of the enhancing intervention.

Since the study subjects were individuals who had consented to participate in the study, a selection bias toward highly motivated individuals was suspected. The overall HPLP II score of the subjects was $2.67 \pm 0.36$ and that of a sample of residents (aged 20-65 years) from the same community [21] was $2.54 \pm 0.37(p<0.05)$, indicating the presence of a selection bias. Nevertheless, the quality of our study was deemed high, given the following background: this study employed a randomly selected control group for comparison with intervention groups; the study subjects were selected from a community in which about $33 \%$ of the population undergo community-based basic or other health checkups [21]; 204 of the 751 (27.2\%) residents who were eligible for enrolment agreed to participate. This percentage is high compared with those reported earlier [22].

The intervention program of this study is characterized by methods that enable program participants to recognize their poor habits by encouraging them to review their lifestyles and, on the basis of a lifestyle survey and health checkup results, set concrete goals for healthier living. The methods also enable participants to reward themselves for achieving the goals they have set in terms of health promotion activities and encourage then to review and reset their goals. Where group health education is employed, program efficacy can be augmented by group dynamism [23]. The above methods are similar to the basic items and methodologies based on Sasaki's behavior modification therapy [24]. The WHO [19] described the necessity of providing both opportunities through which individuals can acquire accurate knowledge and individualized techniques to improve their own lifestyles and programs that meet the various needs of individuals while respecting their independence. Within this context, the programs reported here can be considered to be appropriate.

We found that even the initiation program was significantly effective in improving the scores of all HPLP-II subscales in the participants whose test results indicated pre-metabolic syndrome. The effectiveness of intervention was indicated not only in PA and $\mathrm{N}$, but also in other subscales, such as HR, SG, and SM, with the exception of IR, although the program focused on PA and N. At the final assessment, we were able to demonstrate that the enhancing program maintained the effectiveness of the lifestyle improvement shown at the interim assessment, demonstrating a more dominant effect in the total than in the initiation group, especially for PA and N. Both groups showed significantly higher scores in all subscales of HPLP-II at the final assessment in compared to baseline. In contrast, the control group showed no difference in the score of each HPLP-II subscale between the baseline and final assessment, again demonstrating the effectiveness of both intervention programs. In the total group, there was a slight decrease in the intervention effect in almost all items at the 1-year-after assessment in comparison with the final assessment; this tendency was not clear in the initiation group. However, both groups showed significantly higher scores in all subscales of HPLP-II at the 1-year-after than 
that at baseline, indicating that the effectiveness of both intervention programs was sustained at the end of the 2-year follow-up. However, the scores in almost subscales of HPLP-II at the 1-year-after assessment were higher in the initiation group than in the total group, although the difference was not significant.

Based on these results, we have drawn two important conclusions that can be used to advance the practice of intervention in the community. One is the difficulty in maintaining improved lifestyles, and the other is a sufficient effectiveness of the initiation program, a much shorter program. In terms of the number of dropouts, it was noticeable that, during the enhancing program, there were more dropouts in the total than in the initiation group, even though the difference was not significant. These results suggested that, as discussed in an earlier study [25], there might be an adverse effect of excessive intervention, suggesting the need for an adequate length of intervention. It was also noticeable that the dropout rate of subjects from the final to the 1-year-after assessment was greater in the initiation group than in the total group, although this difference was also not significant.

The same effectiveness of lifestyle improvement was noted in the results of laboratory test data in the follow-up. On the other hand, the BMI in the two groups decreased at the end of the initiation program compared with baseline, and this effect was still evident at the end of the 2-year follow-up, suggesting that it might reflect the effect of a sustained improvement in lifestyle. As reported by Suka et al. [9], the results demonstrate the effectiveness and validity of intervention in the pre-metabolic phase.

Overall, our results demonstrate that the two series of health support programs we have developed for self-oriented improvement of the lifestyle were effective for community residents with some health abnormalities related to metabolic syndrome. In the near future, we should develop more compact and short-term programs that primarily focus on introducing the contents of the initiation program. As reported in an earlier study [26], there are a number of limitations in such intervention programs aimed at promoting the self-improvement of an individual's lifestyle by only applying the health support program. To the residents and staff of Town A, we should emphasize the importance of arranging the living environment, such as promoting the use of health-related institutions, of places for training, and of equipment, and introducing effective systems to facilitate community health professionals in their role of encouraging the residents of their community to practice healthy behavioral habits acquired through a health support program.

Acknowledgments We would like to extend our deep appreciation to the staff of the Health and Welfare Section of Town A Municipal
Office, Kumamoto Prefecture, Japan, for their support in our study. This study was undertaken as a part of a national model project ("Health-Up" model projects of National Health Insurance) by the Japanese Ministry of Health, Labour and Welfare.

\section{References}

1. National Center for Health Statistics. Advance report of final mortality statistics. National Center for Health Statistics, Tokyo. 1990.

2. McGinnis JM, Foege WH. Actual causes of death in the United States. JAMA. 1993;270:2207-12.

3. World Health Organization (WHO). The world health report 2002_reducing risks promoting healthy life. Geneva:World Health Organization; 2002.

4. Health and Welfare Statistics Association. J Health Welf Stat. 2005;141 (in Japanese).

5. Reaven GM. Banting lecture 1988. Role of insulin resistance in human disease. Diabetes. 1988;37:1595-607.

6. Kaplan NM. The deadly quartet: upper-body obesity, glucose intolerance, hypertriglyceridemia, and hypertension. Arch Intern Med. 1989;149:1514-20.

7. Matsuzawa Y. Pathophysiology and molecular mechanisms of visceral fat syndrome: the Japanese experience. Diabetes Metab Rev. 1997;13:3-13.

8. Suka M, Sugimori Y, Yoshida K. MRFS; multiple risk factor syndrome. Health Sci. 2000;16:188-200.

9. Suka M, Yoshida K. An epidemiological approach to the metabolic syndrome (in Japanese). Jpn J Public Health. 2004;51(8): 623-30.

10. Berkman LF, Breslow L. Health and ways of living: the Alameda County Study. Oxford: Oxford University Press; 1983.

11. Morimoto K, editor. Lifestyle and health: introduction (in Japanese). Tokyo: Igaku-shoin; 1991, p 4.

12. WHO Regional Office for Europe. Ottawa Charter for health promotion. Geneva: WHO; 1986.

13. Pender NJ. Health promotion in nursing practice. 3rd edn. Upper Saddle River: Prentice Hall; 1996.

14. Walker SN, Sechrist KR, Pender NJ. The health-promoting lifestyle profile: development and psychometric characteristics. Nurs Res. 1987;36:76-81.

15. Wei CN, Yonemitsu H, Harada K, Miyakita T, Omori S, Ueda A, et al. A Japanese language version of the health-promoting lifestyle profile (in Japanese). Jpn J Hyg. 2000;54(4):597-606.

16. Walker SN, Volkan K, Sechrist KR, Pender NJ. Health-promoting life styles of older adults: comparisons with young and middle-aged adults, correlates and patterns. Adv Nurs Sci. 1988;11(10):76-90.

17. Ennis M, Thain J, Boggild M, Baker GA, Young CA. A randomized controlled trial of a health promotion education program for people with multiple sclerosis. Clin Rehabil. 2006;20(9):783-92.

18. Armitage P, Berry G. Statistical methods in medical research. Oxford: Blackwell; 1994.

19. World Health Organization (WHO). New approaches to health education in primary health care. Geneva: WHO; 1983.

20. Stephen B, Steven R, Warren S, Deborah G, Norman H, Thomas B. Designing clinical research an epidemiologic approach second edition. Philadelphia: Lippincott Williams \& Wilkins; 2001.

21. Ueki machi, Nursing care insurance and Welfare plan and Healthy Japan 21 in Ueki town. 2006; pp 18, pp 48. (in Japanese).

22. Egawa K, Oida Y, Arao T, Matsuzuki H, Shirako M, Kasai W. Efficacy of a community-based weight reduction program to improve exercise and diet behavior in overweight adults (in Japanese). Jpn J Public Health. 2007;54(12):847-56. 
23. Karen G, Barbara K, Frances M. Health behavior and health education; theory, research and practice, 3rd edn. London: Wiley; 2002.

24. Sasaki A. Management of lifestyle related disease-behavior therapy for obesity (in Japanese). Jpn J Behav Med Jpn Soc Behav Med. 2001;7(2):74-82.
25. Rollnick S, editor. Health behavior change. Philadelphia: Harcourt Health Sciences; 1999.

26. Okamoto A. Background and the problem of "Health up" model projects of the National Health Insurance (in Japanese). Public Health. 2007;71(3):206-9. 